\title{
A Novel Method for Driving Path Planning with Spark
}

This paper was downloaded from TechRxiv (https://www.techrxiv.org).

\section{LICENSE}

CC BY 4.0

SUBMISSION DATE / POSTED DATE

$26-08-2021$ / 29-08-2021

CITATION

Lin, Hao (2021): A Novel Method for Driving Path Planning with Spark. TechRxiv. Preprint. https://doi.org/10.36227/techrxiv.16443846.v1

$\mathrm{DOI}$

10.36227/techrxiv.16443846.v1 


\title{
A Novel Method for Driving Path Planning with Spark
}

\author{
Leixiao LI ${ }^{1,3}$, Hao LIN ${ }^{2}$, Jianxiong Wan ${ }^{1,3}$, Yongsheng Wang ${ }^{1,3}$, Jing GAO $O^{4,5}$ \\ 1. College of Data Science and Application, Inner Mongolia University of Technology, China; \\ 2. School of Computer Science and Engineering, Tianjin University of Technology, China; \\ 3. Inner Mongolia Autonomous Region Engineering and Technology Research Center of Big Data \\ Based Software Service, China; \\ 4. College of Computer and Information Engineering, Inner Mongolia Agricultural University, China; \\ 5. Inner Mongolia Autonomous Region Key Laboratory of big data research and application for \\ agriculture and animal husbandry, China
}

\begin{abstract}
Efficient and accurate driving path planning can help drivers drive. To solve the problem of low efficiency of traditional heuristic algorithms such as PSO and GA in solving driving path planning, we introduce Excellence Coefficient into heuristic algorithms and make a parallel design based on Spark, which called EC-SPPSOGA. Excellence Coefficient can increase the probability of good edges being left, simultaneously, preserves the possibility of longer side being selected. The parallel design is based on time-consuming analysis of heuristic algorithms. We validate the performance of EC-SPPSOGA based on the data in TSPLIB. It is verified that the EC-SPPSOGA can improve efficiency of driving path planning and has good scalability.
\end{abstract}

Key words: driving path planning, heuristic algorithms, excellence coefficient, Spark

\section{Introduction}

Driving path planning can provide the optimal driving route for travelers, which is of great significance to alleviating traffic congestion, improving the efficiency of citizens' travel, and improving the happiness index of residents ${ }^{[1][2]}$. The core of driving path planning is the shortest path problem, which can be solved by the TSP problem solving method. TSP is a shortest path problem that has been widely studied, which is also a NP-hard problem ${ }^{[3]}$.

Because of the high computational complexity, traditional methods including Dynamic Programming (DP) and Branch-And-Bound cannot solve TSP. Heuristic algorithms are the mainstream algorithms to solve TSP ${ }^{[4]}$. Hanif et al. ${ }^{[5]}$ focused on six heuristic algorithms: Nearest Neighbor (NN), Genetic Algorithm (GA), Simulated Annealing (SA), Tabu Search (TS), Ant Colony Optimization (ACO) and Tree Physiology Optimization (TPO) to solve TSP problem towards a satisfactory solution. The result shows that TS and ACO take longer than other algorithms. GA can find better solution for Large-scale TSP followed by TPO and SA. Zhang et al ${ }^{[6]}$ proposed a hybrid algorithm called PSO-GA which consists of Particle Swarm Optimization (PSO) and GA to solve TSP. For dynamic TSP, Lukasz et al. ${ }^{[7]}$ proposed a self-adaptive discrete PSO. Result shows that the proposed algorithm outperforms PSO and ACO. But none of the above researches considered the efficiency of heuristic algorithms. The efficiency of Heuristic algorithms is better than traditional methods such as DP, which still has room for improvement.

In addition, because some roads may be short but very congested, driving path planning should

Inner Mongolia Key Technological Development Program (2019ZD015，2019ZD016), Key Scientific and Technological Research Program of Inner Mongolia Autonomous Region (2019GG273), Inner Mongolia Autonomous Region Special Program for Engineering Application of Scientific and Technical Payoffs（2020CG0073）, Key Technologies R\&D Program of Inner Mongolia Autonomous Region（Big Data Resource System and Application Research of Ecological Environment Monitoring, 2020GG0094) 
also provide the optimal path based on the road conditions in the future. For the above problem, we combine our and other's previous researches ${ }^{[8]-[9]}$ to propose a parallel driving path planning solution based on Time Optimal TSP (TOTSP). TOTSP is a variation problem of TSP which replace the length of sides with the time which walk across the sides ${ }^{[10]}$.

Specifically, our contributions are as follows:

- We combine our previous researches to make the parallel design for PSO and GA to solve driving path planning based on TOTSP in parallel.

- We combine literature [11] to introduce Excellence Coefficient (EC) into PSO and GA to improve solution accuracy. The algorithm is named EC-SPPSOGA by us.

- In the scene of driving route analysis, we validate the performance of EC-SPPSOGA based on the real data of Hohhot and the data in TSPLIB.

\section{Formal Description}

The target of TSP is to find the shortest closed path which traverses all target locations only once. Figure 1 illustrates the Graph network for TSP.

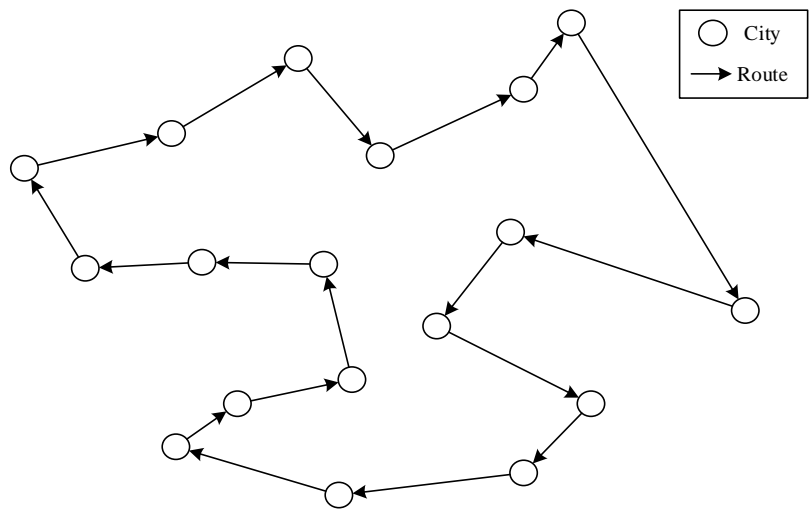

Fig.1 Graph network for TSP

Let $G(N, E)$ is a connected graph, where $N$ means vertex set or city set and $V$ means the set of distances between two vertices or cities. Set $N=\{1,2, \cdots, H\} \quad V=\left\{\left(i, j, w_{i j}\right), i, j\right.$ Í $\left.N, w_{i j} i^{+}\right\}$, Hamilton loop path that traverses all vertices once is as follows

$$
\text { Hamilton }=\left\{V_{k_{1} k_{2}}, V_{k_{2} k_{3}}, \cdots, V_{k_{H} k_{1}}\right\}, k_{j} \mid \begin{aligned}
& \text { I } \\
& \text { H }
\end{aligned} k_{i}, k_{j} \text { Ú } j \text {. }
$$

Objective function of TSP is as follows

$$
\begin{aligned}
& \min Z=\stackrel{a ̈}{a}_{i, j=1}^{n} x_{i j} w_{i j} \\
& x_{i j} \mid\{\{0,1\}, i, j \neq, 2, \cdots, n \text {. }
\end{aligned}
$$

Where $x_{i j}$ is the decision variable. When the traveling salesman goes from $i$ to $j, x_{i j}=1$. Otherwise $x_{i j}=0$.

The target of TOTSP is not to find the shortest path, but to find the path that takes the least time. Let $S$ the set of speed between two vertices or cities and $S=\left\{\left(i, j, s_{i j}\right), i, j\left\{i, s_{i j} \quad R^{+}\right\}\right.$. Objective function of TOTSP is as follows

$$
\begin{gathered}
\min Z={\stackrel{a ̈}{a} x_{i j} \frac{w_{i j}}{s_{i j}}}_{x_{i j} \mid\{\quad\{0,1\}, i, j \neq, 2, \cdots, n .}
\end{gathered}
$$




\section{EC-SPPSOGA Method}

The basis of EC-SPPSOGA proposed in this paper is a hybrid algorithm of GA and PSO, which is shown in Fig.2.

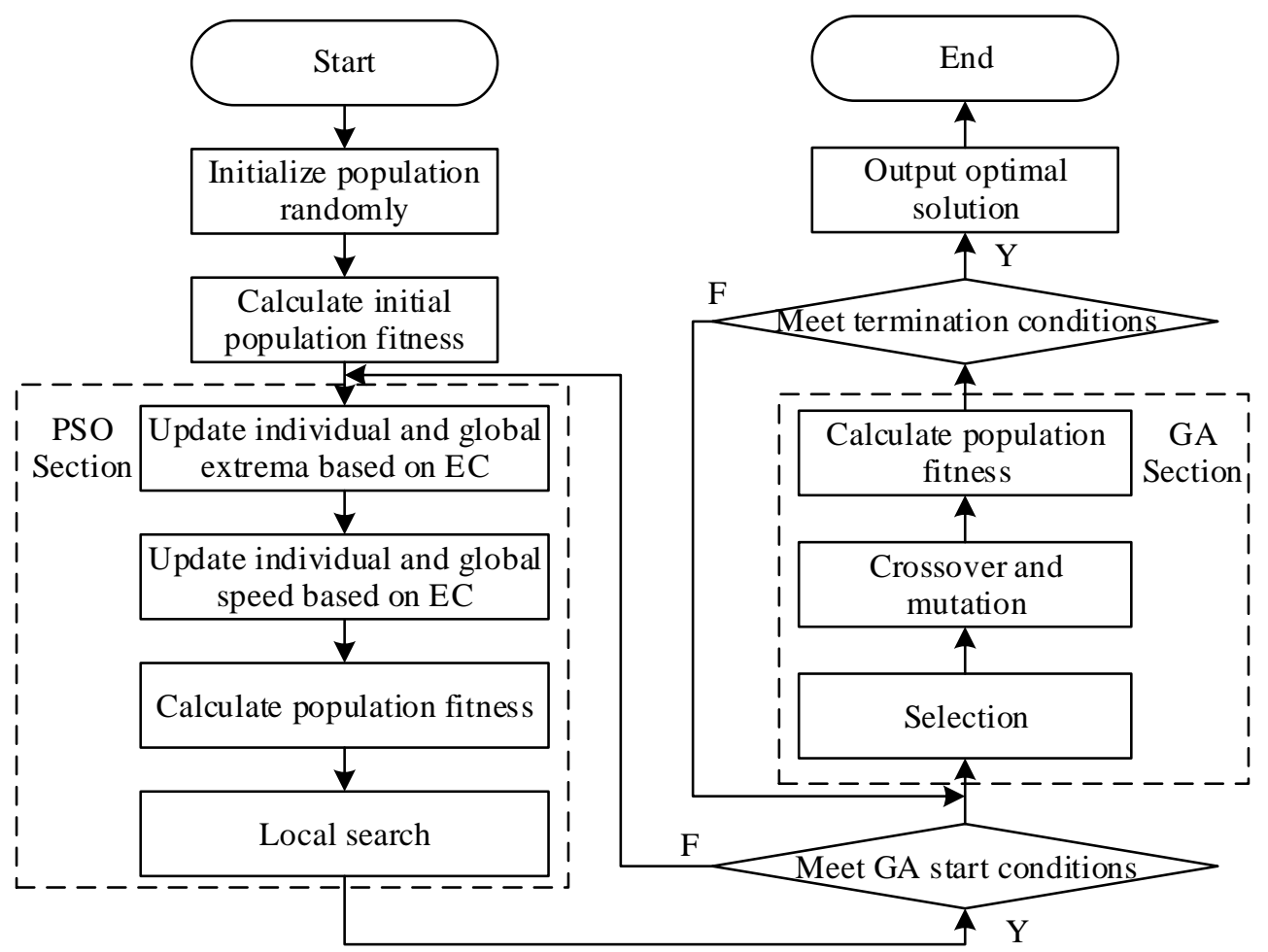

Fig.2 Hybrid algorithm of GA and PSO proposed in this paper

\subsection{PSO Section}

We introduce EC into traditional PSO to improve performance of PSO. In actual, most of the edges of the optimal path are the line between a certain point $a$ and one of the nearest points to $a$. In TOTSP, most of the edges of the optimal path that takes the least time are the line between a certain city $n$ and one of the nearest cities to $n$. Therefore, to improve the convergence performance of PSO, the probability of the short edge being selected should be appropriately increased. EC is designed for this problem. EC $C_{i j}$ is defined as

$$
C_{i j}=\frac{C_{i j} \mathrm{i}}{\max \left(C_{i j} \mathrm{i}\right)} .
$$

Where $C_{i j} \mathrm{i}$ is defined as

$$
C_{i j} \mathrm{i}=\frac{\text { @̀max }(d)-d_{i j}}{\text { ä } d_{i j}} \text {. }
$$

Where $d_{i j}$ means the distances between $i$ and $j$. The shorter the edge corresponds to the larger $C_{i j} \mathbf{i}$ and the larger $C_{i j}$. Introduce $C_{i j}$ into PSO can increase the probability of good edges being left. Simultaneously, $C_{i j}$ preserves the possibility of longer side being selected. 
After the introduction of EC, the particle velocity and position update formula of PSO are as follow

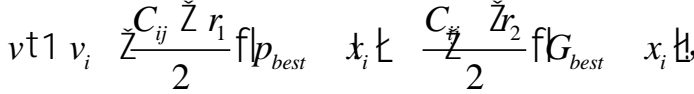

$$
\begin{aligned}
& x \mathrm{i}=x_{i} \quad \boldsymbol{t}_{i} .
\end{aligned}
$$

Where $v$ i means updated particle speed; $x$ i means updated particle position; $v_{i}$ means current particle velocity; $x_{i}$ means current particle position; $r_{1}$ and $r_{2}$ mean random number in $(0,1) ; p_{\text {best }}$ means individual extremum; $G_{b e s t}$ means global extremum. The addition of the three parts in Eq.(6) reflects the sharing of information between particles. Arithmetic means of $C_{i j}, r$ incorporates EC into PSO and ensures the overall randomness of the heuristic algorithm ${ }^{[12]}$.

\subsection{Local Search}

In TOTSP, the solution with path crossing must not be the optimal solution. Because crossover of feasible solutions, heuristic algorithms perform poorly in solving large-scale TOTSP ${ }^{[13]}$. Through the triangle property that the sum length of any two sides is greater than the length of third side, we can prove that the solution after eliminating the cross path must be better than the solution with the cross path.

Set the length of the two sides are $l(i, i+1)$ and $l(j, j+1)$. If satisfied

$$
l(i, i+1)+l(j, j+1) \quad l i, j) \quad l(\not+1, j+1),
$$

we can think that the two sides intersect. To optimize the solution, the original two sides should be deleted and side $<i, j>i<1,+1 \cdot$ should be linked. The idea of local search is shown in Fig.3.

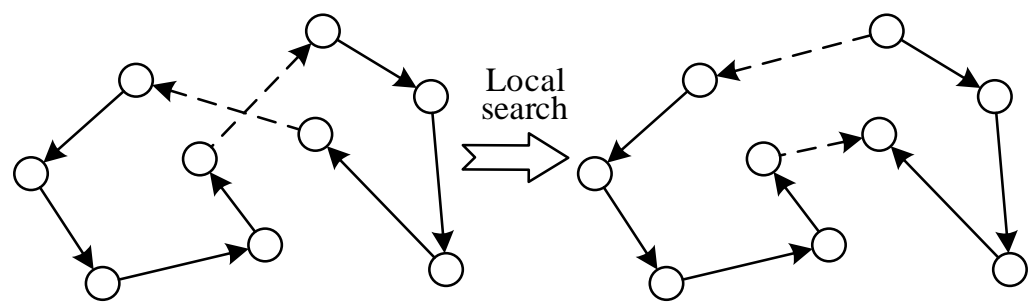

Fig.3 Local search strategy that eliminates crossover

\subsection{GA Section}

GA is responsible for further optimize the population optimized by PSO. Roulette is used to select inherited individuals in GA section. The fitness function of GA is as follow

$$
\text { fitness }=1 / Z \quad \neq / \ddot{a ̆}_{i, j=1}^{n} x_{i j} \frac{w_{i j}}{s_{i j}} \text {. }
$$

Where $Z$ is the same as $Z$ in Eq.(3) (i.e., the reciprocal of the total path length is used as the fitness function of GA).

Due to selection of GA is similar to EC, we only introduce EC into PSO. Simultaneously, due to crossover and mutation of GA are similar to local search, we do not introduce local search into GA.

\subsection{Parallel Design}

The algorithm shown in Fig.2 can be divided into three part including population initiation, population update, fitness calculation. Fig.4 illustrates the divided structure. 


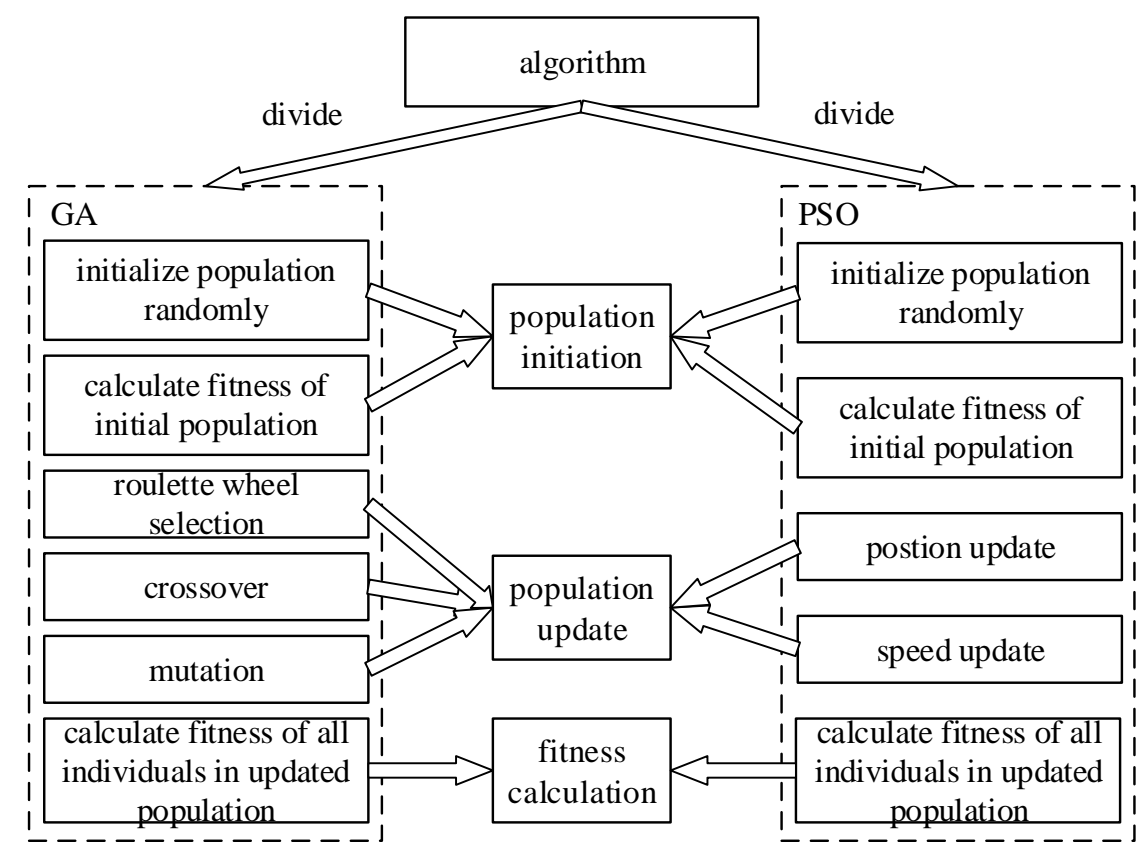

Fig.4 Divided structure of algorithm shown in Fig.2

As Fig. 4 shows that population initiation includes two parts-initialize the population randomly and calculate fitness of the initial population. Population update includes five parts-roulette wheel selection, crossover, mutation, speed update and position update. Fitness calculation is responsible for calculating the fitness of all individuals in the updated population.

We run the algorithm shown in Fig.2 20 times to record time spent by three parts. Where the maximum number of iterations is 1000 and population size is 300. The results are shown in Fig.5.

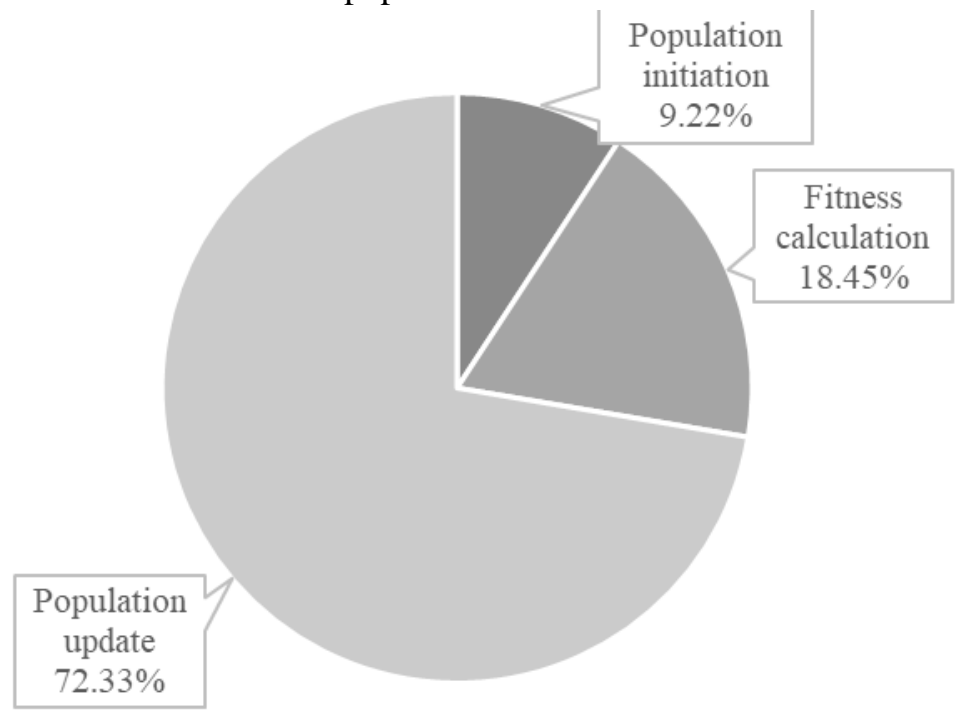

Fig.5 Time-consuming of three parts

As Fig. 5 shows that the time spent by population update accounted for $72.33 \%$ of the total time. The time spent by population initiation only accounted for $9.22 \%$ of the total time. Based on the result, we use Spark to parallelize the algorithm shown in Fig.2 to reduce time spent by population initiation and fitness calculation. We call the parallelized algorithm EC-SPPSOGA, which is shown in Fig.6. 


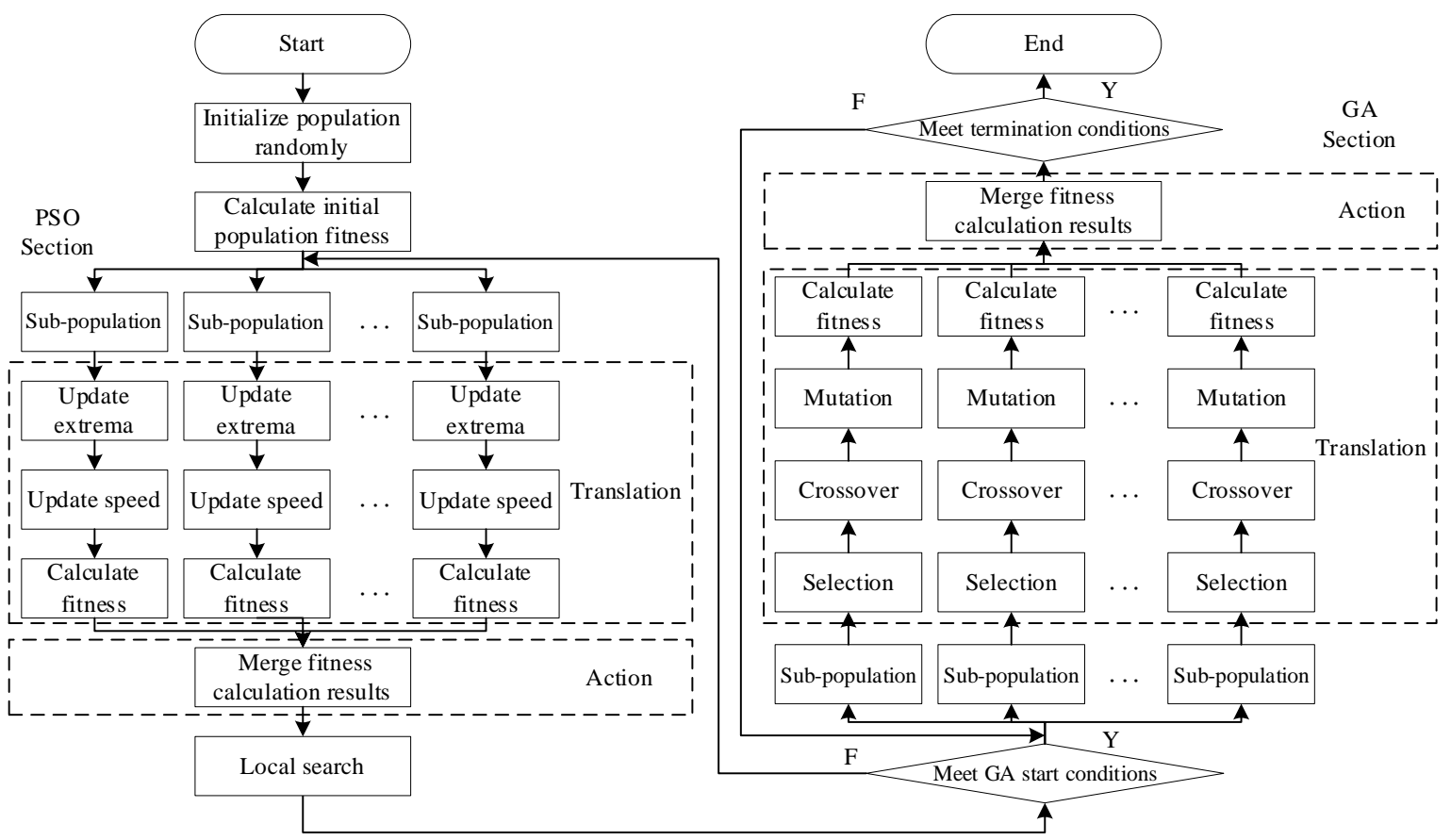

Fig.6 Flow process figure of EC-SPPSOGA

Parallel design is based on the special data format Resilient Distributed Datasets (RDD) in Spark. RDD supports two operations including the operation called Translation which can generate a new RDD and the operation called Action which can calculate the content in the RDD to return the result ${ }^{[14]}$. In PSO section of EC-SPPSOGA, we use map function to update the extreme value and velocity of particles in parallel and calculate individual fitness in parallel. Because lazy computing of RDD, collect function is used to trigger the calculations involved in map function to merge fitness calculation results. In GA section of EC-SPPSOGA, we use map function to carry out operations such as selection, crossover, and mutation in parallel. Finally, terminate the iteration of the algorithm when the termination condition is met.

\section{Performance Evaluation and Result Analysis}

\subsection{Experimental Data and Parameter Setting}

We use real latitude and longitude data of Hohhot to build vertex set. To accurately calculate the driving distance, we take the latitude and longitude data to six decimal places. Part of the data is shown in Table 1.

Table1 Some longitude and latitude data of intersections

\begin{tabular}{cccc}
\hline Number & Intersection name & longitude & latitude \\
\hline 1 & Aimin Street - Zhelimu Road & 111.687455 & 40.849464 \\
2 & Aimin Street - Xing'an North Road & 111.698486 & 40.851838 \\
3 & Xing'an North Road -Kangfu Road & 111.695181 & 40.861033 \\
$\ldots$ & $\ldots$ & $\ldots$ & $\ldots$ \\
24 & Saimachang North Road - Tiyuguan East Lane & 111.671989 & 40.858013 \\
\hline
\end{tabular}

We collect the speed data of the roads involved in these intersections at 18:00 on December 11 to simulate road conditions in the future.

We implement EC-SPPSOGA using python and carry out experiment in an environment where the CPU is $17-8700 @ 3.2 \mathrm{G}$ and the RAM is 16G. The number of experimental tests is 50 times. The 
parameter settings are shown in Table 2.

Table2 Parameter settings of EC-SPPSOGA related experiments

\begin{tabular}{ccccccccc}
\hline Algorithm & $\begin{array}{c}\text { Population } \\
\text { size }\end{array}$ & $\begin{array}{c}\text { Maximum } \\
\text { iteration }^{\circledR}\end{array}$ & $\begin{array}{c}\text { Crossover } \\
\text { probability }\end{array}$ & $\begin{array}{c}\text { Mutation } \\
\text { probability }\end{array}$ & $\begin{array}{c}\text { Information } \\
\text { factor }\end{array}$ & $\begin{array}{c}\text { Heuristic } \\
\text { factor }\end{array}$ & $\begin{array}{c}\text { Information } \\
\text { enhancement } \\
\text { factor }\end{array}$ & $\begin{array}{c}\text { Pheromone } \\
\text { evaporation } \\
\text { coefficient }\end{array}$ \\
\hline GA & 100 & 200 & 0.9 & 0.3 & N/A & N/A & N/A & N/A \\
PSO & 100 & 200 & N/A & N/A & N/A & N/A & N/A & N/A \\
ACO & 100 & 200 & N/A & N/A & 1 & 5 & 100 & 0.1 \\
\hline
\end{tabular}

\subsection{Performance Experiment}

The experiment is responsible for proving the feasibility of the driving route decision analysis method based on the road conditions in the future and verifying the performance of EC-SPPSOGA in various data sets. We use GA, ACO and SECPSO as the comparison algorithm of EC-SPPSOGA, and use the data in Table 1 to optimize the driving route. Experimental results are shown in Table 3.

Table3 Experimental results of driving path planning by different calculation methods

\begin{tabular}{ccccccccc}
\hline \multirow{2}{*}{ Algorithm } & \multicolumn{3}{c}{ Path length-based (TSP-based) } & \multicolumn{5}{c}{ Future road conditions-based (TOTSP-based) } \\
\cline { 2 - 9 } & K-Opt & Opt & Ave & Sta & K-Opt & Opt & Ave & Sta \\
\hline ACO & 14.55 & 14.7247 & 16.3882 & 0.8224 & 14.4104 & 14.6832 & 16.3396 & 0.7808 \\
GA & 14.55 & 14.55 & 14.5802 & 0.0288 & 14.4104 & 14.4104 & 14.4383 & 0.0532 \\
SECPSO & $\mathbf{1 4 . 5 5}$ & $\mathbf{1 4 . 5 5}$ & $\mathbf{1 4 . 5 5}$ & $\mathbf{0}$ & $\mathbf{1 4 . 4 1 0 4}$ & $\mathbf{1 4 . 4 1 0 4}$ & $\mathbf{1 4 . 4 1 0 4}$ & $\mathbf{0}$ \\
EC-SPPSOGA & $\mathbf{1 4 . 5 5}$ & $\mathbf{1 4 . 5 5}$ & $\mathbf{1 4 . 5 5}$ & $\mathbf{0}$ & $\mathbf{1 4 . 4 1 0 4}$ & $\mathbf{1 4 . 4 1 0 4}$ & $\mathbf{1 4 . 4 1 0 4}$ & $\mathbf{0}$ \\
\hline
\end{tabular}

${ }^{\mathrm{a}}$ K-Opt represents the known optimal solution; Opt represents the optimal value; Ave represents the average value; Sta represents the standard deviation

The optimal solution based on path length and the optimal solution based on future road conditions at 18:00 on December 11 are shown in Fig.7.
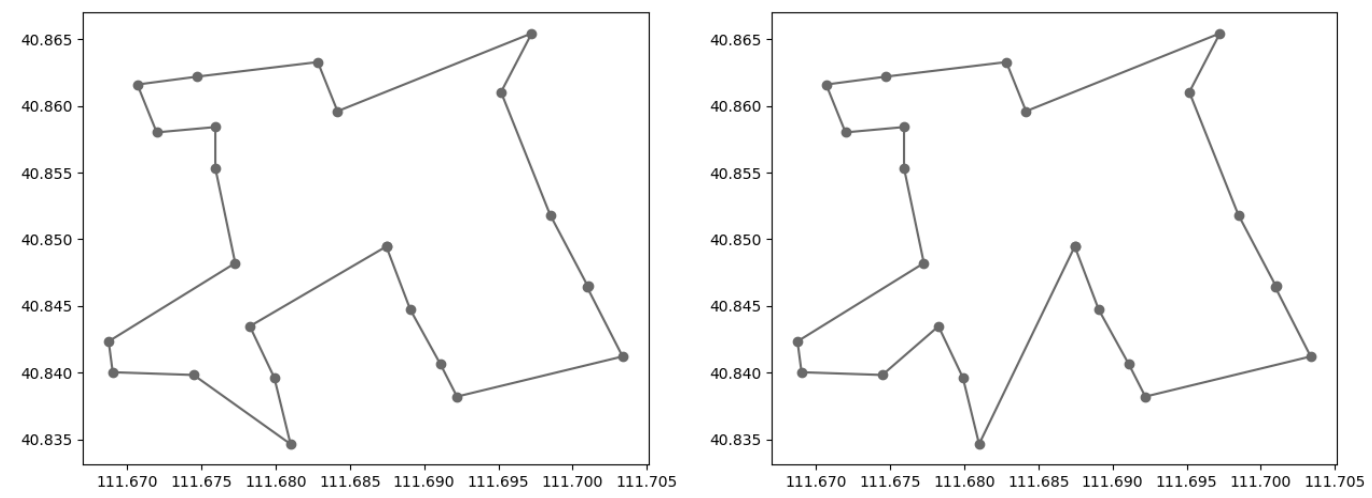

(a) Optimal solution based on path length (b) Optimal solution based on future road conditions Fig.7 Optimal solution result display

The results in Table 3 illustrate the optimal solution based on future road conditions is better than the optimal solution based on path length. It proves the feasibility of the TOTSP-based driving path planning method. The performance of SECPSO and EC-SPPSOGA is significantly better than that of ACO and GA. But the performance difference between SECPSO and EC-SPPSOGA needs further verification.

For further verifying the performance of EC-SPPSOGA, we use the data sets in TSPLIB to carry out performance experiment. The selected data set has more vertexes than the data set shown in Table 1 . New experimental results are shown in Table 4.

\footnotetext{
(1) The maximum number of iterations is 200 , if GA and PSO are mixed, each iteration will be 100 times.
} 
Table4 Experimental results using TSPLIB and VLSITSP data sets

\begin{tabular}{cccccccccccccc}
\hline Data & \multirow{2}{*}{\begin{tabular}{c}
\multirow{2}{*}{ Ket } \\
K-Opt
\end{tabular}} & \multicolumn{3}{c}{ ACO } & \multicolumn{3}{c}{ GA } & \multicolumn{4}{c}{ SECPSO } & \multicolumn{3}{c}{ EC-SPPSOGA } \\
\cline { 2 - 13 } & Opt & Ave & Sta & Opt & Ave & Sta & Opt & Ave & Sta & Opt & Ave & Sta \\
\hline eil76 & 538 & 563 & 609 & 20.2 & 569 & 608 & 19.09 & 538 & 586 & 17.48 & $\mathbf{5 3 8}$ & $\mathbf{5 7 7}$ & $\mathbf{1 3 . 1 7}$ \\
ch130 & 6110 & 6556 & 6676 & 78.77 & 6504 & 6700 & 78.99 & 6380 & 6461 & 56.28 & $\mathbf{6 3 1 1}$ & $\mathbf{6 3 9 1}$ & $\mathbf{5 3 . 7 8}$ \\
gr202 & 550 & 555 & 627.9 & 54.65 & 553 & 623.4 & 49.14 & 550 & 565.1 & 9.72 & $\mathbf{5 5 0}$ & $\mathbf{5 6 3 . 6}$ & $\mathbf{7 . 8 5}$ \\
\hline
\end{tabular}

The results in Table.4 illustrate, for a multi-vertex data set, GA's performance is slightly better than ACO's. In addition, the optimal value and average value of EC-SPPSOGA are similar to SECPSO, but the standard deviation of EC-SPPSOGA is smaller than that of SECPSO. The results show that driving route optimization using EC-SPPSOGA is more stable. In both simple and complex data set, EC-SPPSOGA is superior to other comparison algorithms.

\subsection{Scalability Experiment}

The experiment is responsible for proving the scalability of EC-SPPSOGA. We run EC-SPPSOGA on single node, 2 nodes, 4 nodes and 8 nodes to record the time-consuming and speedup using data shown in Table 1. The speedup is calculated as

$$
S_{n}=\frac{T_{1}}{T_{n}} .
$$

Where $T_{1}$ means the algorithm time-consuming on a single node. $T_{n}$ means the algorithm time-consuming on $n$ nodes.

Since the population size determines the computation of EC-SPPSOGA, we use the population size and the number of Spark cluster nodes as the independent variables of the experiment. The experimental results are shown in Fig.8.

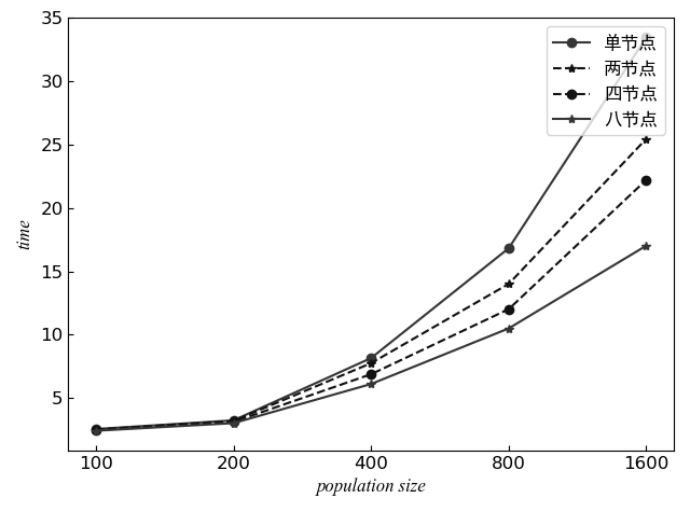

(a) Training time results under different computations

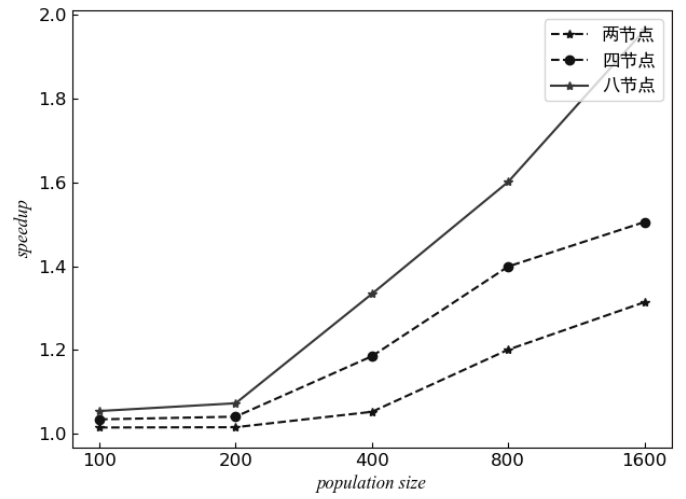

(b) Speedup calculation results under different computations

Fig.8 Scalability experiment results

As Fig.8(a) shows that, as the computation gradually increases, the running time gradually increases. When the computation is small, the training efficiency of the algorithm on single node, 2 nodes, 4 nodes, and 8 nodes are not much different. As the computation increases, the running time of 8 nodes will be much lower than that of 4 nodes, 2 nodes and single node.

The conclusion from Fig.8(b) is similar to that from Fig.8(a). When the computation is small, speedup is not higher than 1.1. The value indicates that the parallel efficiency is not good. As the computation increases, the parallel efficiency is getting higher and higher. Because of speedup of 8 nodes is less than 2.0, the acceleration effect is not significant.

The problem is related to the number of vertexes in the data set. So, we use data set ch130 (it has 130 vertexes) to rerun the experiment. New experimental results are shown in Fig.9. 


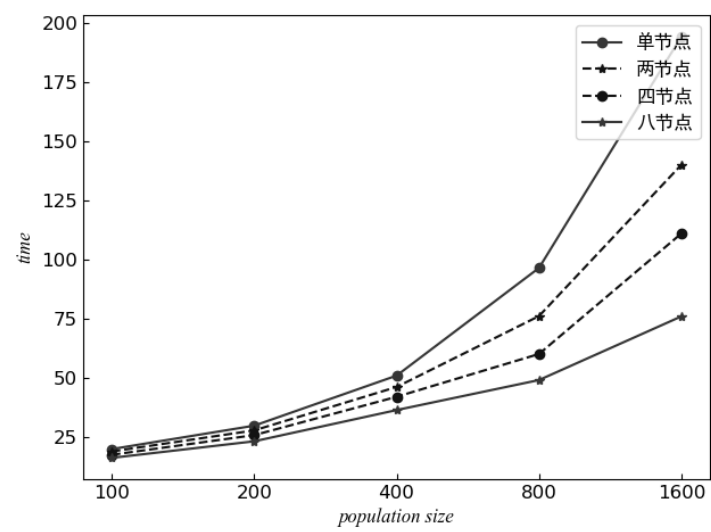

(a) Train time with different calculation

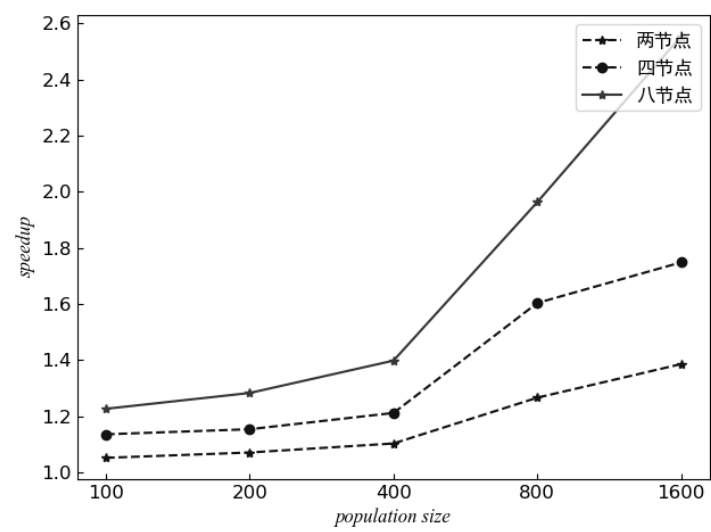

(b) Speedup with different calculation

Fig.9 Scalability experiment results in case of 130 vertexes

As Fig.9(b) shows that, when the population size is 1600 and the number of iterations is 200, speedup of the EC-SPPSOGA on 8 nodes can reach 2.6. The experimental results prove that the greater computation, the more obvious acceleration effect. Simultaneously, it is verified that the EC-SPPSOGA proposed in this paper has good scalability.

\section{Conclusion}

We proposed a new algorithm for driving path planning based on PSO and GA. The algorithm is introduced EC and parallel design by Spark to improve efficiency. We use the data in TSPLIB to experiment and verified that the algorithm can improve efficiency of driving path planning and has good scalability.

\section{References}

[1] Wang Y, Zhao J J, Li X J, et al. 'Review of vehicle route planning based on traffic big data', Software guide,2020,19(10),pp.50-54.

[2] Li J, Yang F. 'Multiple task object traversal path planning in complex obstacle environment', Modern Electronics Technique,2021,44(07),pp.162-168.

[3] Ebadinezhad S. 'DEACO: Adopting dynamic evaporation strategy to enhance ACO algorithm for the traveling salesman problem', Engineering Applications of Artificial Intelligence, 2020, 92,pp.103649.

[4] Thomas W, Yan J, Qi Q, et al. 'A branch-and-bound-based crossover operator for the traveling salesman problem', International Journal of Cognitive Informatics and Natural Intelligence, 2019,13(3),pp.1-18.

[5] Hanif H A., Ismail I. 'Combinatorial optimization: comparison of heuristic algorithms in travelling salesman problem', Archives of Computational Methods in Engineering, 2017, 26(2), pp. 367-380.

[6] Zhang Y, Chen L, Xu X L, et al. 'Research on time optimal TSP based on hybrid PSO-GA', Application Research of Computers, 2015,32(12),pp.3613-3617.

[7] Lukasz S, Rafał S, Urszula B, et al. 'A self-adaptive discrete PSO algorithm with heterogeneous parameter values for dynamic TSP', Entropy, 2019,21(8), pp.738.

[8] Li L X, Lin H, Wan J, et al. 'MF-TCPV: a machine learning and fuzzy comprehensive evaluation-based framework for traffic congestion prediction and visualization', IEEE Access, 2020, 8, pp. 227113-227125.

[9] Lin H, Li X L, Wang H. 'SPGAPSO-SVM: a prediction algorithm of urban bus passenger flow', Journal of Chinese Computer Systems ,2020,41(11), pp.2458-2464. 
[10]Ting L, Zhu J M. 'The application of multi-objective TOTSP in scenic spot tour route planning', International Journal of Intelligent Information and Management Science, 2019,8(3), pp.91-93.

[11]Cheng B Y, Lu H Y, Xu X P, et al. 'Improved local-search-based chaotic discrete particle swarm optimization algorithm for solving traveling salesman problem', Journal of Computer Applications, 2016,36(01),pp.138-142+149.

[12]Cheng B Y, Lu H Y, Huang Y, et al. 'Particle swarm optimization algorithm based on self-adaptive excellence coefficients for solving traveling salesman problem', Journal of Computer Applications, 2017,37(3), pp.750-754+781.

[13]Jaszkiewicz A. 'Genetic local search for multi-objective combinatorial optimization', European Journal of Operational Research, 2002, 137(1), pp.50-71.

[14]Huang. C Q, Yang S Q, Tang J C, et al. 'RDDShare: reusing results of spark RDD', Proceedings of 1st DSC. Changsha, China, 2016, pp.370-375. 